\title{
Tyrosine kinase inhibitors for the therapy of anaplastic thyroid cancer
}

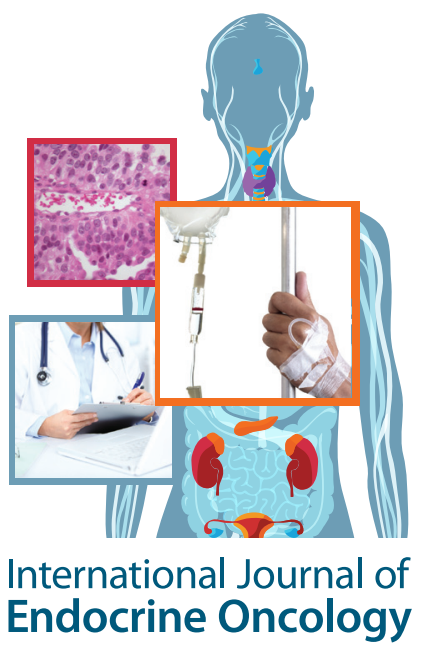

Anaplastic thyroid cancer (ATC) is often incurable so new therapeutic approaches are needed. Tyrosine kinases inhibitors (such as imanitib, sunitinib or sorafenib) are under evaluation for the treatment of ATC. Other vascular disrupting agents, such as combretastatin A4 phosphate, and antiangiogenic agents, such as aplidin, PTK787/ ZK222584 and human VEGF monoclonal antibodies (bevacizumab, cetuximab), have been evaluated. Small-molecule adenosine triphosphate competitive inhibitors directed intracellularly at EGFRs tyrosine kinase, such as erlotinib or gefitinib, are also studied. Furthermore, new molecules have been shown to be active against ATC, such as CLM94 and CLM3. However, more research is needed to finally identify therapies able to control and to cure this disease.

Keywords: anaplastic thyroid cancer $\bullet$ BRAFV600E • combination therapy and salvage therapy $\bullet$ EGF $\bullet$ PPAR- $\gamma$ agonists $\bullet$ RAS $\bullet$ targeted therapies $\bullet$ tyrosine kinase inhibitors $\bullet$ VEGF

Anaplastic thyroid cancer (ATC) has a very poor prognosis due to its aggressive behavior and resistance to cancer treatments [1].

Surgery followed by chemotherapy and radiotherapy can significantly prolong the survival of patients carrying ATC intrathyroidal tumors, but this kind of presentation is very unusual. In fact, ATC is often advanced and metastatic at diagnosis [2,3]. Currently, the most effective treatment of ATC is multimodal treatment protocol that includes surgery, chemotherapy (doxorubicin and cisplatin) and hyperfractionated accelerated external beam radiotherapy with a median patient survival of 10 months [4].

During the last two decades, several somatic mutations in different molecular pathways of anaplastic thyroid carcinomas have been revealed and associated with progression of ATC $[5,6]$.

Thus, clinical research targeting these pathways has been recently explored. There are a number of clinical trials for anaplastic thyroid carcinoma underway or being planned using different types of drugs, such as peroxisome proliferator-activated receptor
(PPAR- $\gamma$ ) agonists, and tyrosine kinase inhibitors [6].

\section{Genetic study \& molecular pathways in ATC}

To date, several genetic mutations have been identified that play roles in the carcinogenesis of ATC. A well-studied mutation of thyroid cancer is BRAFV600E, occurring in approximately $26 \%$ of ATCs and $45 \%$ of papillary thyroid cancers (PTCs) $[7,8]$.

The tumor suppressor gene $\mathrm{p} 53$ is frequently mutated in ATC [7] but uncommon in well-differentiated PTC and follicular thyroid cancer (FTC) (with a frequence ranging from 71 to $88 \%$ in ATC) [9,10]. Point mutations within RAS genes involve codons NRAS, HRAS and KRAS, with mutations of NRAS and HRAS at codon 61 and of KRAS at codon 12/13 being the most common. Mutant RAS proteins activate the MAPK and PI3K/AKT pathways. RAS mutations are found in $\sim 10-15 \%$ PTCs, $40-50 \%$ of FTCs and in $\sim 35 \%$ of poorly differentiated and $\sim 50 \%$ of ATCs, where the presence of RAS mutations seems to

\author{
Alessandro Antonelli*,1, \\ Poupak Fallahi', Salvatore \\ Ulisse², Silvia Martina \\ Ferrari', Valeria Mazzi', \\ Andrea Di Domenicantonio' \\ \& Paolo Miccoli $^{3}$ \\ 'Department of Clinical \& Experimental \\ Medicine, University of Pisa, Via Savi, 10, \\ I-56126 Pisa, Italy \\ ${ }^{2}$ Department of Experimental Medicine, \\ Sapienza University of Rome, \\ Viale dell'Università, 30, \\ I-00185 Rome, Italy \\ ${ }^{3}$ Department of Surgical, Medical, \\ Molecular Pathology \& Critical Area, \\ University of Pisa, Via Savi, 10 , \\ I-56126 Pisa, Italy \\ *Author for correspondence: \\ Tel.: +39050992318 \\ Fax: +39050 553235 \\ alessandro.antonelli@med.unipi.it
}


correlate with a more aggressive tumor behavior and poor prognosis [11,12].

Mutations of PIK3CA are also common in ATC [7]. PIK3CA encodes the p110 $\alpha$ catalytic subunit of PI3K and PI3K, which regulates cell cycle progression, adhesion and motility [13]. Garcia-Rostan et al. [13] showed that the missense mutations of PIK3CA are frequently present in ATC.

VEGF-A is the major mediator of tumor angiogenesis, promoting the proliferation and survival of endothelial cells and increasing vascular permeability [14]. Differentiated thyroid cancer (DTC) has been found to express high levels of both VEGF, because of upregulation of its main receptor, VEGFR-2, with respect to normal thyroid [15]. Moreover, increased expression of VEGF in thyroid cancer has been associated with an increase in tumor size, distant metastasis and poor prognosis [16].

EGFR (ErbB-1; HER1 in humans) is the cell-surface receptor for members of the epidermal growth factor family (EGF-family) [17]. Mutations, amplifications or misregulations of EGFR or family members are implicated in about $30 \%$ of all epithelial cancers. EGFR is overexpressed in ATC, and is implicated in tumor progression and invasion in thyroid cancer $[18,19]$.

Liu et al. [20] found frequent copy number gains in many receptor tyrosine kinase genes containing the EGFR, PDGFR- $\alpha$, PDGFR- $\beta$, VEGFR1, VEGFR2, KIT, MET, PIK3Ca, PIK3Cb and PDK1 genes. Many of the genes play an important role in ATC tumorigenesis by aberrant activation of the oncogenic pathway. The prevalence of copy number gains was generally higher in ATC than in DTC [21]. It is suggested that the copy number variations should be important and involved in the progression and aggressiveness of ATC [22].

A major mechanism controlling cellular differentiation and biological behavior of cancer cells is histone acetylation that results in a more open chromatin configuration that increases the gene transcription rate. Cancer cells have been found to have dysregulated histone acetyltransferase or histone deacetylase activity [23,24].

It has been reported [20] RET/PTC rearrangement in three cases of ATC tissues, probably because some ATC tissues contained PTC tissues.

More recently, it has been suggested the upregulated expression of miR-20a in ATC counteracts thyroid cancer progression and may have therapeutic potential [25].

Many papers have recently reviewed the comprehensive genetic alterations in ATC to identify genomic alterations associated with ATC [6].

\section{PPAR- $\gamma$ agonists}

PPAR $-\gamma$ are members of a superfamily of nuclear hormone receptors [26]; activation of PPAR- $\gamma$ isoforms elicits antineoplastic [27] effects in several types of cells. Recently, it has been shown in vitro that activating ligands of PPAR- $\gamma$ : induce apoptosis and exert antiproliferative effects on human PTC cells [26]; prevent distant metastasis of BHP18-21 tumors in nude mice in vivo [26] and induce redifferentiation in thyroid cancer [28-30]. The expressions of the PPAR- $\gamma$ are increased in human ATC cell lines [31], with respect to PTC, and PPAR- $\gamma$ ligands inhibit proliferation and invasion, and induce apoptosis [3133]. Rosiglitazone was able to increase the expression of thyroid-specific differentiation markers, suggesting that PPAR- $\gamma$ agonists induce a partial reversion of the epithelial mesenchymal transition in ATC cells [32]. Furthermore, it has been recently shown in 'primary cultured cells from human ATC' (ANA), obtained from each patient $[34,35]$, that PPAR- $\gamma$ agonists rosiglitazone and pioglitazone are able to inhibit cell growth.

On the basis of the encouraging results of in vitro studies, recently an in vivo trial has been done.

Recently a novel high-affinity PPAR- $\gamma$ agonist (RS5444), which is dependent upon PPAR- $\gamma$ for its biological activity, has been shown to inhibit proliferation of $\left(\mathrm{IC}_{50} \sim 0.8 \mathrm{nM}\right)$ ATC cells, and of ATC tumor in nude mice [36].

It was also demonstrated that reactivation of suppressed RhoB is a critical step for the inhibition of ATC growth [33].

More recently, a Phase I study evaluated efatutazone and paclitaxel in ATC. Fifteen ATC patients received efatutazone $(0.15,0.3$ or $0.5 \mathrm{mg})$ orally twice daily and then paclitaxel every 3 weeks. Median times to progression were 48 and 68 days in patients receiving $0.15 \mathrm{mg}$ of efatutazone and $0.3 \mathrm{mg}$ of efatutazone, respectively; corresponding median survival was 98 versus 138 days. Eight patients had $\geq 1$ serious adverse event. The authors suggest that efatutazone and paclitaxel in combination were safe and tolerated and had biologic activity [37].

\section{Targeted therapy for ATC \\ Raf kinase pathway}

The orally active multikinase inhibitor (mKI) sorafenib, targeting VEGFR-1 and -2, B-Raf, RET and c-Kit, is a potentially effective agent for patients with thyroid cancer, due to its effects on RET, the B-Raf pathway and angiogenesis. Several Phase II clinical trials, and one Phase III trial, have evaluated the use of sorafenib in patients with metastatic iodine refractory thyroid carcinoma [38-41]. 
The results of these studies suggest that sorafenib is a new treatment option for patients with progressive radioactive iodine-refractory DTC [41].

Patients with ATC who had failed up to previous therapies were enrolled in a multi-institutional Phase II trial of sorafenib. Twenty of them were treated with sorafenib $400 \mathrm{mg}$ twice daily, and 2/20 (10\%) patients had a partial response (PR) and 5/20 (25\%) had stable disease (SD). The overall median progression-free survival (PFS) was 1.9 months, the median and a 1-year survival being 3.9 months and $20 \%$, respectively. The authors conclude that sorafenib is active in ATC even if at a low frequency [42].

\section{EGFR pathway}

The small-molecule EGFR-TK inhibitor gefitinib inhibits cell growth in thyroid cancer lines and in RET-transfected cell lines at submicromolar concentrations [43]. The EGFR kinase inactivation induced by gefitinib potentiates the ionizing radiation-induced inhibition of cell proliferation on FTC and anaplastic cell lines [44].

A Phase II trial was conducted in patients with advanced or metastatic thyroid cancer (DTC $=18$, medullary thyroid cancer $[\mathrm{MTC}]=4$, etc.) who received gefitinib $(250 \mathrm{mg} /$ daily), and reported tumor volume reductions in $32 \%$ of cases (none of them met criteria for PR); $48 \%$ of them attained SD at 3 months; the overall survival (OS) and median PFS were 17.5 months (70 weeks) and 3.7 months (14.8 weeks), respectively, suggesting that gefitinib could not have clinically significant activity as monotherapy [45].

In a patient with ATC treated with an intermittent high-dose gefitinib, and fixed-dose docetaxel, a PR was shown [46].

\section{VEGF pathway}

Vandetanib

The orally bioavailable mKI vandetanib targets EGFR, VEGFR-2 and -3, and RET kinases, and is approved for MTC treatment because of its effects on both RET activation and angiogenesis [47]. Two Phase II trials, and one Phase III trial, on vandetanib have been conducted in patients with MTC with positive results [47-49], and the US FDA and EMA-approved vandetanib [50] for the treatment of aggressive MTC.

A pretherapeutic drug evaluation by tumor xenografting in ATC has been recently published suggesting that vandetanib reduced the tumor volume (up to $61 \%$ ) and tumor vascularity accompanied by decreased EGF-R/VEGF-R2 receptor activity [51].

\section{Axitinib}

The mKI axitinib targets PDGFR, VEGFR-1, -2 and -3 and c-Kit, has great selectivity against VEGFR-2, and is considered the most potent VEGFR-2 inhibitor available. A strong activity of axitinib against thyroid cancer was evidenced in a Phase II trial on 60 patients with advanced, iodine-refractory thyroid cancer using axitinib $5 \mathrm{mg}$ b.i.d. [52]. PR was shown in 18 patients (30\%; eight patients with PTC, six FTC, two MTC and one ATC). Moreover, SD was also observed in other 23 patients (38\%) and median PFS was 18.1 months (72.4 weeks).

Long-term outcomes were evaluated in a recent study in 60 patients with advanced thyroid cancer treated with axtinib. Objective response rate was 38\% (23 patients had PR, and 18 had SD lasting $\geq 16$ weeks). Responses occurred in all histologic subtypes. The median follow-up was of 34 months, median OS was 35 months, median PFS was 15 months and median duration of response was 21 months. These results suggest that axitinib is active and well tolerated in patients with advanced thyroid cancer demonstrating long OS [53].

\section{Sunitinib}

The mKI sunitinib targets c-Kit, VEGFR-2, PDGFR, RET, FLT-3 and CSF-1R [54]. Two Phase II trials with sunitinib in thyroid cancer have been published [55,56].

Recently, the results of a large open-label Phase II trial, which included 28 patients with progressive DTC and 7 patients with MTC [57] have been presented. They showed complete response in one patient, PR in $28 \%$ and SD in $46 \%$ of patients [57].

Recently a paper reported a case report showing clinical and visual activity using sunitinib as a salvage treatment in an ATC patient who was not fit to receive systemic chemotherapy treatment [58]. After the end of the second cycle, 12 weeks from the beginning of sunitinib treatment, a complete macroscopic response of the ATC in the neck was achieved. However, response in tumor size over neck mass did not correlate with lung metastasis shrinkage that were stable. Unfortunately, the patient died as a result of a massive upper gastrointestinal bleeding 5 months after the start of sunitinib treatment and while the patient was still on treatment [58].

\section{Lenvatinib (E7080)}

Lenvatinib (E7080) is an oral inhibitor of PDGFRb, VEGFR-1, -2, -3 RET, FGFR-1, -2, -3, -4 and c-KIT, it has been shown active in advanced DTC [59].

The antitumor activity of lenvatinib against human thyroid cancer was evaluated in xenograft models in nude mice (five DTC, five ATC and one MTC). Lenvatinib showed antiangiogenesis activity in DTC and in ATC xenografts [60]. 


\section{CLM94, CLM3}

Antonelli et al. demonstrated the antitumoral activity of CLM94, a novel cyclic amide with VEGFR-2 and antiangiogenic activity, in ANA cells both in vitro and in vivo [6].

CLM3 (a mKI that inhibits RET tyrosine kinase, EGFR, VEGFR2 and with antiangiogenic activity) $[62,63]$ can inhibit in vitro the proliferation of ANA, inducing apoptosis. CLM3, significantly inhibited EGFR, AKT and ERK $1 \frac{1}{2}$ phosphorylation, and cyclin D1, and decreased the VEGF-A expression and microvessel density in ANA. The above-mentioned data showed the antitumor and antiangiogenic activity of CLM3 is very promising in ATC, opening a future avenue to clinical evaluation [63].

\section{Vascular disrupting mechanism \\ Combretastatin}

The microtubule depolymerizing agent combretastatin A4 phosphate (CA4P) exerts selectively its activity against established tumor vascular networks, producing interruption of tumor blood flow and necrosis of the tumor tissue [64]. A complete response was reported in a Phase I trial conducted on ATC, in one patient treated with combretastatin, and was alive 30 months after treatment [65].

The FACT trial was a randomized, controlled Phase II/III trial assessing the safety and efficacy of carboplatin/paclitaxel with CA4P (experimental arm) or without CA4P (control arm) in ATC [66]. A total of 80 patients were enrolled; $55 \%$ had undergone a cancer-related operation, of whom $70 \%$ had neartotal/total thyroidectomy. Baseline characteristics for operative and nonoperative patients were not substantially different. Median survival for patients who had cancer-related operation was 8.2 months in the CA4P arm versus 4.0 months in the control arm. This suggests that thyroidectomy followed by CA4P combination regimen shows a nonsignificant trend toward improvement in patient survival [66].

An open-label study of doublet carboplatin/paclitaxel chemotherapy with or without fosbretabulin in patients with ATC has been recently conducted. Eighty patients were enrolled. There was no significant difference in PFS between the two arms [67].

\section{Targeted therapies resistance}

Clinical experience suggests that many of treatmentresponsive patients experience relapse as a result of acquired resistance to targeted therapies [68]. This resistance is often associated with genomic changes (such as the amplification of a completely different cancer gene, or an additional point mutation within the gene encoding the protein to which the drug is targeted) originally present in minimal subclones of cancer cells [69]. Thus, the production of second-generation drugs to combat resistance is clinically important. For example, in chronic myeloid leukemia patients resistance to imatinib therapy is associated with secondary mutations within the Abl kinase domain. Reduced drug sensitivity seems to be conferred by all of these mutations; second-generation Abl inhibitors (such as nilotinib and dasatinib) [70] can bypass the resistance of the imatinib-refractory $\mathrm{Abl}$ mutations, showing significant clinical activity.

The use of combination strategies that could minimize the possibilities of the resistant clones ever expanding have been evaluated, too.

For the above-mentioned reasons, the identification of new active compounds against aggressive DTC is needed [61].

\section{Combination therapy \& salvage therapy}

Many clinical studies have indicated several limitations to the application of TKIs as a single agent in various types of cancers. For these reasons, the potential of sorafenib (or other TKIs) to synergize with other targeted agents, or chemotherapy, or radiation, has been widely explored with promising results [71-73].

In a clinical trial [74], sorafenib, in combination with tipifarnib (a farnesyltransferase inhibitor that inactivates Ras), was given to 35 patients with DTC and MTC in a Phase I trial. MTC PR rate was $38 \%$ (5 of 13), SD of at least 6 months was $31 \%$. The DTC PR rate was $4.5 \%$, and SD of at least 6 months was 36\%. Median PFS for all 35 patients was 18 months. The authors concluded that inhibiting the Ras/Raf/MAPK kinase/ERK and RET kinase pathways with sorafenib and tipifarnib is well tolerated and active against thyroid cancer.

The role of salvage therapy was recently evaluated in 64 patients with metastatic DTC who received salvage therapy after their initial sorafenib failure. Salvage therapy included sunitinib $(n=4)$, pazopanib $(n=3)$, cabozantinib $(n=4)$, lenvatinib $(n=3)$ and vemurafenib $(n=3)$. Median OS of all 64 patients receiving first-line sorafenib was 37 months; median OS was significantly longer with salvage therapy compared with sorafenib alone (58 vs 28 months). Median PFS was 7.4 months with first-line sorafenib and 11.4 months with salvage therapy. These results suggest that other targeted agents are effective salvage treatments after sorafenib failure, despite similar mechanisms of action, and should be offered to patients who are able to receive salvage therapy [75].

\section{Personalization of therapy}

The advent of not expensive individual genomic analysis could lead to a new era of patient-specific, personalized care. Moreover, in vitro drug screening 
in primary human tumor cells [76] can lead to a negative predictive value of $90 \%$, and a positive predictive value of $60 \%$ [77] for the activity of clinical responses, allowing to avoid the administration of inactive chemotherapeutics to patients [78].

Until now, primary thyroid cancer cell cultures have been obtained from surgical biopsies. Fine-needle aspiration (FNA) cytology by passes the need of surgery. The possibility to obtain 'primary cell culture directly from FNA cytology samples of ATC' (FNAANA) paves the way to the use of FNA-ANA to test the sensitivity in each patient to different drugs. This could avoid unnecessary surgical procedures and the administration of inactive therapeutics $[34,35,79,80]$.

\section{Conclusion \& future perspective}

Different alterations of genes and molecular pathways have been shown in ATC (B-Raf, and VEGFR-2, EGFR, etc.). The development of novel compounds that target genes and molecular pathways playing a crucial role in the development of ATC has led to the introduction of new drugs that might overcome in the future the lack of effective therapies for ATC. However, until now, no significant amelioration of survival in ATC patients has been shown with targeted therapies. Furthermore, resistance and 'escape' to TKIs treatments have been described.
The potential of TKIs to synergize with other targeted agents, or chemotherapy, or radiation has been widely explored with promising results, to overcome the resistance to a single TKI agent. Furthermore, it has been shown that other targeted agents might be effective salvage treatments after a first-line TKI failure, despite similar mechanisms of action.

To reach the goals to extend life duration assuring a good quality of life, the identification of new compounds is needed. Furthermore, the advent of not expensive individual genomic analysis could lead to a new era of patient-specific, personalized care. Moreover, the personalization of the treatment could be achieved testing these novel drugs in primary ATC cells (obtained from each patient) in vitro to avoid the administration of inactive therapeutics.

\section{Financial \& competing interests disclosure}

The authors have no relevant affiliations or financial involvement with any organization or entity with a financial interest in or financial conflict with the subject matter or materials discussed in the manuscript. This includes employment, consultancies, honoraria, stock ownership or options, expert testimony, grants or patents received or pending, or royalties.

No writing assistance was utilized in the production of this manuscript.

\section{Executive summary}

- Anaplastic thyroid cancer (ATC) has a very poor prognosis due to its aggressive behavior and resistance to cancer treatments.

- Currently, the most effective treatment of ATC is multimodal treatment protocol including surgery, chemotherapy (doxorubicin and cisplatin) and hyperfractionated accelerated external beam radiotherapy (median patient survival of 10 months).

Genetic study and molecular pathways in ATC

- Different alterations of genes and molecular pathways have been shown in ATC (B-Raf and VEGFR-2, EGFR, etc.).

- The development of novel compounds that target genes and molecular pathways playing a crucial role in the development of ATC has led to the introduction of new drugs that might overcome in the future the lack of effective therapies for ATC.

Targeted therapy for ATC

- Tyrosine kinases inhibitors (such as imanitib, sunitinib or sorafenib) are under evaluation for the treatment of ATC; antiangiogenic agents, vascular disrupting agents, such as combretastatin A4 phosphate, and smallmolecule adenosine triphosphate competitive inhibitors directed intracellularly at EGFRs tyrosine kinase, such as gefitinib, have been evaluated.

Targeted therapy for ATC

- To reach the goals to extend life duration assuring a good quality of life, the identification of new compounds is needed.

\section{Personalization of therapy}

- The advent of not expensive individual genomic analysis could lead to a new era of patient-specific, personalized care.

\section{Personalization of therapy}

- The possibility to test these novel drugs in primary ATC cells (obtained from each patient) in vitro could help improve the personalization of the treatment, avoiding the administration of inactive therapeutics. 


\section{References}

Papers of special note have been highlighted as:

•• of considerable interest

1 Are C, Shaha AR. Anaplastic thyroid carcinoma: biology, pathogenesis, prognostic factors, and treatment approaches. Ann. Surg. Oncol. 13(4), 453-464 (2006).

2 Miccoli P, Materazzi G, Antonelli A et al. New trends in the treatment of undifferentiated carcinomas of the thyroid. Langenbecks Arch. Surg. 392(4), 397-404 (2007).

- 3 Kebebew E. Anaplastic thyroid cancer: rare, fatal, and neglected. Surgery 152(6), 1088-1089 (2012).

4 De Crevoisier R, Baudin E, Bachelot A et al. Combined treatment of anaplastic thyroid carcinoma with surgery, chemotherapy, and hyperfractionated accelerated external radiotherapy. Int. J. Radiat. Oncol. Biol. Phys. 60(4), 1137-1143 (2004).

-5 Antonelli A, Fallahi P, Ferrari SM et al. New targeted therapies for thyroid cancer. Curr. Genomics 12(8), 626-631 (2011).

-6 Smallridge RC, Ain KB, Asa SL et al. American Thyroid Association guidelines for management of patients with anaplastic thyroid cancer. Thyroid 22(11), 1104-1139 (2012).

-. The recomandation of ATA for management of patients with anaplastic thyroid cancer (ATC).

7 Smallridge RC, Marlow LA, Copland JA. Anaplastic thyroid cancer: molecular pathogenesis and emerging therapies. Endocr. Relat. Cancer 16(1), 17-44 (2009).

-8 Xing M. BRAF mutation in thyroid cancer. Endocr. Relat. Cancer 12(2), 245-262 (2005).

- Fagin JA, Matsuo K, Karmakar A et al. High prevalence of mutations of the $\mathrm{p} 53$ gene in poorly differentiated human thyroid carcinomas. J. Clin. Invest. 91(1), 179-184 (1993).

- 10 Quiros RM, Ding HG, Gattuso P, Prinz RA, Xu X. Evidence that one subset of anaplastic thyroid carcinomas are derived from papillary carcinomas due to BRAF and $\mathrm{p} 53$ mutations. Cancer 103(11), 2261-2268 (2005).

-11 Nikiforova MN, Nikiforov YE. Molecular genetics of thyroid cancer: implications for diagnosis, treatment and prognosis. Expert Rev. Mol. Diagn. 8(1), 83-95 (2008).

-12 Ruggeri RM, Campennì A, Baldari S, Trimarchi F, Trovato M. What is New on Thyroid Cancer Biomarkers. Biomark. Insights 3, 237-252 (2008).

-13 Garcia-Rostan G, Costa AM, Pereira-Castro I et al. Mutation of the PIK3CA gene in anaplastic thyroid cancer. Cancer Res. 65(22), 10199-10207 (2005).

14 Ferrara N. Vascular endothelial growth factor: basic science and clinical progress. Endocr. Rev. 25(4), 581-611 (2004).

15 Bunone G, Vigneri P, Mariani L et al. Expression of angiogenesis stimulators and inhibitors in human thyroid tumors and correlation with clinical pathological features. Am. J. Pathol. 155(6), 1967-1976 (1999).

16 Lennard CM, Patel A, Wilson J et al. Intensity of vascular endothelial growth factor expression is associated with increased risk of recurrence and decreased disease-free survival in papillary thyroid cancer. Surgery 129(5), 552-558 (2001).
17 Herbst RS. Review of epidermal growth factor receptor biology. Int. J. Radiat. Oncol. Biol. Phys. 59(2 Suppl.), 21-26 (2004).

18 Knauf JA. Does the epidermal growth factor receptor play a role in the progression of thyroid cancer? Thyroid 21(11), 1171-1174 (2011).

19 Yeh MW, Rougier JP, Park JW et al. Differentiated thyroid cancer cell invasion is regulated through epidermal growth factor receptor-dependent activation of matrix metalloproteinase (MMP)-2/gelatinase A. Endocr. Relat. Cancer 13(4), 1173-1183 (2006).

-20 Liu Z, Hou P, Ji M et al. Highly prevalent genetic alterations in receptor tyrosine kinases and phosphatidylinositol 3-kinase/akt and mitogen-activated protein kinase pathways in anaplastic and follicular thyroid cancers. J. Clin. Endocrinol. Metab. 93(8), 3106-3116 (2008).

21 Bond JA, Wyllie FS, Rowson J, Radulescu A, WynfordThomas D. In vitro reconstruction of tumour initiation in a human epithelium. Oncogene 9(1), 281-290 (1994).

-22 Hou P, Liu D, Shan Y et al. Genetic alterations and their relationship in the phosphatidylinositol 3-kinase/ Akt pathway in thyroid cancer. Clin. Cancer Res. 13(4), 1161-1170 (2007).

23 Mahlknecht U, Hoelzer D. Histone acetylation modifiers in the pathogenesis of malignant disease. Mol. Med. 6(8), 623-644 (2000).

24 Marks P, Rifkind RA, Richon VM et al. Histone deacetylases and cancer: causes and therapies. Nat. Rev. Cancer 1(3), 194-202 (2001).

25 Xiong Y, Zhang L, Kebebew E. MiR-20a is upregulated in anaplastic thyroid cancer and targets LIMK1. PLoS ONE 9(5), e96103 (2014).

Ohta K, Endo T, Haraguchi K, Hershman JM, Onaya T. Ligands for peroxisome proliferator-activated receptor gamma inhibit growth and induce apoptosis of human papillary thyroid carcinoma cells. J. Clin. Endocrinol. Metab. 86(5), 2170-2177 (2001).

Grommes C, Landreth GE, Heneka MT. Antineoplastic effects of peroxisome proliferator-activated receptor gamma agonists. Lancet Oncol. 5(7), 419-429 (2004).

28 Klopper JP, Hays WR, Sharma V et al. Retinoid X receptorgamma and peroxisome proliferator-activated receptorgamma expression predicts thyroid carcinoma cell response to retinoid and thiazolidinedione treatment. Mol. Cancer Ther. 3(8), 1011-1020 (2004).

29 Park JW, Zarnegar R, Kanauchi H et al. Troglitazone, the peroxisome proliferator-activated receptor-gamma agonist, induces antiproliferation and redifferentiation in human thyroid cancer cell lines. Thyroid 15(3), 222-231 (2005).

30 Fröhlich E, Machicao F, Wahl R. Action of thiazolidinediones on differentiation, proliferation and apoptosis of normal and transformed thyrocytes in culture. Endocr. Relat. Cancer 12(2), 291-303 (2005).

- 31 Hayashi N, Nakamori S, Hiraoka N et al. Antitumor effects of peroxisome proliferator activate receptor gamma ligands on anaplastic thyroid carcinoma. Int. J. Oncol. 24(1), 89-95 (2004). 
- 32 Aiello A, Pandini G, Frasca F et al. Peroxisomal proliferatoractivated receptor-gamma agonists induce partial reversion of epithelial-mesenchymal transition in anaplastic thyroid cancer cells. Endocrinology 147(9), 4463-4475 (2006).

33 Marlow LA, Reynolds LA, Cleland AS et al. Reactivation of suppressed $\mathrm{RhoB}$ is a critical step for the inhibition of anaplastic thyroid cancer growth. Cancer Res. 69(4), 1536-1544 (2009).

34 Antonelli A, Ferrari SM, Fallahi P et al. Thiazolidinediones and antiblastics in primary human anaplastic thyroid cancer cells. Clin. Endocrinol. (Oxf). 70 (6), 946-953 (2009).

- 35 Antonelli A, Fallahi P, Ferrari SM et al. Dedifferentiated thyroid cancer: a therapeutic challenge. Biomed. Pharmacother. 62(8), 559-563 (2008).

36 Copland JA, Marlow LA, Kurakata S et al. Novel highaffinity PPARgamma agonist alone and in combination with paclitaxel inhibits human anaplastic thyroid carcinoma tumor growth via p21WAF1/CIP1. Oncogene 25(16), 2304-2317 (2006).

- 37 Smallridge RC, Copland JA, Brose MS et al. Efatutazone, an oral PPAR- $\gamma$ agonist, in combination with paclitaxel in anaplastic thyroid cancer: results of a multicenter phase 1 trial. J. Clin. Endocrinol. Metab. 98(6), 2392-2400 (2013).

- 38 Kloos RT, Ringel MD, Knopp MV et al. Phase II trial of sorafenib in metastatic thyroid cancer. J. Clin. Oncol. 27(10), 1675-1684 (2009).

- 39 Gupta-Abramson V, Troxel AB, Nellore A et al. Phase II trial of sorafenib in advanced thyroid cancer. J. Clin. Oncol. 26(29), 4714-4719 (2008).

- 40 Hoftijzer H, Heemstra KA, Morreau H et al. Beneficial effects of sorafenib on tumor progression, but not on radioiodine uptake, in patients with differentiated thyroid carcinoma. Eur. J. Endocrinol. 161(6), 923-931 (2009).

41 Brose MS, Nutting CM, Jarzab B et al. Sorafenib in radioactive iodine-refractory, locally advanced or metastatic differentiated thyroid cancer: a randomised, double-blind, phase 3 trial. Lancet 384(9940), 319-328 (2014).

-. The first Phase III trial of sorafenib in radioactive iodinerefractory, locally advanced or metastatic differentiated thyroid cancer.

-42 Savvides P, Nagaiah G, Lavertu P et al. Phase II trial of sorafenib in patients with advanced anaplastic carcinoma of the thyroid. Thyroid 23(5), 600-604 (2013).

43 Croyle M, Akeno N, Knauf JA et al. RET/PTC-induced cell growth is mediated in part by epidermal growth factor receptor (EGFR) activation: evidence for molecular and functional interactions between RET and EGFR. Cancer Res. 68(11), 4183-4191 (2008).

44 Lopez JP, Wang-Rodriguez J, Chang CY et al. Gefitinib (Iressa) potentiates the effect of ionizing radiation in thyroid cancer cell lines. Laryngoscope 118(8), 1372-1376 (2008).

45 Pennell NA, Daniels GH, Haddad RI et al. A phase II study of gefitinib in patients with advanced thyroid cancer. Thyroid 18(3), 317-323 (2008).

-• The Phase II trial of gefitinib in ATC.

46 Fury MG, Solit DB, Su YB et al. A phase I trial of intermittent high-dose gefitinib and fixed-dose docetaxel in patients with advanced solid tumors. Cancer Chemother. Pharmacol. 59(4), 467-475 (2007).

\$47 Wells SA Jr, Gosnell JE, Gagel RF et al. Vandetanib for the treatment of patients with locally advanced or metastatic hereditary medullary thyroid cancer. J. Clin. Oncol. 28(5), 767-772 (2010).

-48 Robinson BG, Paz-Ares L, Krebs A, Vasselli J, Haddad R. Vandetanib $(100 \mathrm{mg})$ in patients with locally advanced or metastatic hereditary medullary thyroid cancer. J. Clin. Endocrinol. Metab. 95(6), 2664-2671 (2010).

49 Wells SA Jr, Robinson BG, Gagel RF et al. Vandetanib in patients with locally advanced or metastatic medullary thyroid cancer: a randomized, double-blind Phase III trial. J. Clin. Oncol. 30(2), 134-141 (2012).

-. The first Phase III trial of vandetanib in medullary thyroid cancer.

50 Thornton K, Kim G, Maher VE et al. Vandetanib for the treatment of symptomatic or progressive medullary thyroid cancer in patients with unresectable locally advanced or metastatic disease: U.S. Food and Drug Administration drug approval summary. Clin. Cancer Res. 18(14), 3722-3730 (2012).

Wunderlich A, Khoruzhyk M, Roth S et al. A pretherapeutic drug evaluation by tumor xenografting in anaplastic thyroid cancer. J. Surg. Res. 185(2), 676-683 (2013).

\$2 Cohen EE, Rosen LS, Vokes EE et al. Axitinib is an active treatment for all histologic subtypes of advanced thyroid cancer: results from a phase II study. J. Clin. Oncol. 26(29), 4708-4713 (2008).

-53 Cohen EE, Tortorici M, Kim S, Ingrosso A, Pithavala YK, Bycott P. A Phase II trial of axitinib in patients with various histologic subtypes of advanced thyroid cancer: long-term outcomes and pharmacokinetic/pharmacodynamic analyses. Cancer Chemother. Pharmacol. 74(6), 1261-1270 (2014).

54 Kim DW, Jo YS, Jung HS et al. An orally administered multitarget tyrosine kinase inhibitor, SU11248, is a novel potent inhibitor of thyroid oncogenic RET/papillary thyroid cancer kinases. J. Clin. Endocrinol. Metab. 91(10), 4070-4076 (2006).

55 Cohen EE, Needles BM, Cullen KJ et al. Phase 2 study of sunitinib in refractory thyroid cancer. Presented at: $44 \mathrm{th}$ American Society of Clinical Oncology Meeting, Chicago, IL, USA, 30 May-3 June 2008.

56 Goulart B, Carr L, Martins RG et al. Phase II study of sunitinib in iodine refractory, well-differentiated thyroid cancer (WDTC) and metastatic medullary thyroid carcinoma (MTC). Presented at: 44th American Society of Clinical Oncology Meeting, Chicago, IL, USA, 30 May-3 June 2008.

57 Carr LL, Mankoff DA, Goulart BH et al. Phase II study of daily sunitinib in FDG-PET-positive, iodine-refractory differentiated thyroid cancer and metastatic medullary carcinoma of the thyroid with functional imaging correlation. Clin. Cancer Res. 16(21), 5260-5268 (2010).

- A Phase II trial of sunitinib in thyroid cancer.

58 Grande E, Capdevila J, Díez JJ, Longo F, Carrato A. A significant response to sunitinib in a patient with anaplastic thyroid carcinoma. J. Res. Med. Sci. 18(7), 623-625 (2013). 
59 Sherman SI, Jarzab B, Cabanillas ME et al. A phase II trial of the multitargeted kinase inhibitor E7080 in advanced radioiodine (RAI)-refractory differentiated thyroid cancer (DTC). Presented at: 47th American Society of Clinical Oncology Meeting, Chicago, IL, USA, 3 June-7 June 2011.

60 Tohyama O, Matsui J, Kodama K et al. Antitumor activity of lenvatinib (e7080): an angiogenesis inhibitor that targets multiple receptor tyrosine kinases in preclinical human thyroid cancer models. J. Thyroid Res. 2014, 638747 (2014).

61 Antonelli A, Bocci G, Motta La C et al. CLM94, a novel cyclic amide with anti-VEGFR-2 and antiangiogenic properties, is active against primary anaplastic thyroid cancer in vitro and in vivo. J. Clin. Endocrinol. Metab. 97(4), E528-E536 (2012).

62 Ferrari SM, Fallahi P, La Motta C et al. Antineoplastic activity of the multitarget tyrosine kinase inhibitors CLM3 and CLM94 in medullary thyroid cancer in vitro. Surgery 156(5), 1167-1176 (2014).

63 Antonelli A, Bocci G, Fallahi P et al. CLM3, a multitarget tyrosine kinase inhibitor with antiangiogenic properties, is active against primary anaplastic thyroid cancer in vitro and in vivo. J. Clin. Endocrinol. Metab. 99(4), E572-E581 (2014).

64 Kanthou C, Tozer GM. Microtubule depolymerizing vascular disrupting agents: novel therapeutic agents for oncology and other pathologies. Int. J. Exp. Pathol. 90 (3), 284-294 (2009).

65 Dowlati A, Robertson K, Cooney M et al. A phase I pharmacokinetic and translational study of the novel vascular targeting agent combretastatin a- 4 phosphate on a singledose intravenous schedule in patients with advanced cancer. Cancer Res. 62(12), 3408-3416 (2002).

66 Sosa JA, Balkissoon J, Lu SP et al. Thyroidectomy followed by fosbretabulin (CA4P) combination regimen appears to suggest improvement in patient survival in anaplastic thyroid cancer. Surgery 152(6), 1078-1087 (2012).

-. A trial of fosbretabulin in an ATC.

67 Sosa JA, Elisei R, Jarzab B et al. Randomized safety and efficacy study of fosbretabulin with paclitaxel/carboplatin against anaplastic thyroid carcinoma. Thyroid 24(2), 232-240 (2014).

68 Engelman JA, Janne PA. Mechanisms of acquired resistance to epidermal growth factor receptor tyrosine kinase inhibitors in non-small cell lung cancer. Clin. Cancer Res. 14(10), 2895-2899 (2008).

69 McDermott U, Downing JR, Stratton MR. Genomics and the continuum of cancer care. N. Engl. J. Med. 364(4), 340-350 (2011).

-. An important paper on resistance to targeted therapies.
70 Kantarjian H, Giles F, Wunderle L et al. Nilotinib in imatinib-resistant CML and Philadelphia chromosomepositive ALL. N. Engl. J. Med. 354(24), 2542-2551 (2006).

71 Ibrahim N, Yu Y, Walsh WR, Yang JL. Molecular targeted therapies for cancer: sorafenib mono-therapy and its combination with other therapies (review). Oncol. Rep. 27(5), 1303-1311 (2012).

72 Koh YW, Shah MH, Agarwal K et al. Sorafenib and Mek inhibition is synergistic in medullary thyroid carcinoma in vitro. Endocr. Relat. Cancer 19(1), 29-38 (2012).

-73 Kandil E, Tsumagari K, Ma J et al. Synergistic inhibition of thyroid cancer by suppressing MAPK/PI3K/AKT pathways. J. Surg. Res. 184(2), 898-906 (2013).

74 Hong DS, Cabanillas ME, Wheler J et al. Inhibition of the Ras/Raf/MEK/ERK and RET kinase pathways with the combination of the multikinase inhibitor sorafenib and the farnesyltransferase inhibitor tipifarnib in medullary and differentiated thyroid malignancies. J. Clin. Endocrinol. Metab. 96(4), 997-1005 (2011).

75 Dadu R, Devine C, Hernandez M et al. Role of salvage targeted therapy in differentiated thyroid cancer patients who failed first-line sorafenib. J. Clin. Endocrinol. Metab. 99(6), 2086-2094 (2014).

76 Newell DR. Flasks, fibres and flanks-pre-clinical tumour models for predicting clinical antitumour activity. $\mathrm{Br}$. J. Cancer. 84(10), 1289-1290 (2001).

77 Schroyens W, Tueni E, Dodion P, Bodecker R, Stoessel F, Klastersky J. Validation of clinical predictive value of in vitro colorimetric chemosensitivity assay in head and neck cancer. Eur. J. Cancer. 26(7), 834-838 (1990).

78 Blumenthal RD, Goldenberg DM. Methods and goals for the use of in vitro and in vivo chemosensitivity testing. Mol. Biotechnol. 35(2), 185-197 (2007).

79 Antonelli A, Ferrari SM, Fallahi P et al. Evaluation of the sensitivity to chemotherapeutics or thiazolidinediones of primary anaplastic thyroid cancer cells obtained by fine-needle aspiration. Eur. J. Endocrinol. 159(3), 283-291 (2008).

- The first study that evaluated the sensitivity to tyrosine kinase inhibitors not on continuous cells lines but in primary dedifferentiated thyroid cancer cells.

80 Antonelli A, Ferrari SM, Fallahi P et al. Primary cell cultures from anaplastic thyroid cancer obtained by fineneedle aspiration used for chemosensitivity tests. Clin. Endocrinol. 69(1), 148-152 (2008).

- The first study that evaluated the sensitivity to drugs of primary ATC cells obtained by fine-needle aspiration. 